\title{
A giant primary hepatic low-grade malignant mesenchymal tumor of the liver in a teenager
}

\author{
Xuewen Zhang ${ }^{1}$, Changjun Jia ${ }^{1}$, Yang Zhao ${ }^{1}$, Chaoliu Dai ${ }^{1}$, Can $\mathrm{Xu}^{2}$ \\ ${ }^{1}$ Department of Hepatobiliary and Splenic Surgery, ${ }^{2}$ Department of Pathology, Shengjing Hospital, China Medical University, Shenyang 110004 , \\ China \\ Correspondence to: Chaoliu Dai, PhD. Department of Hepatobiliary and Splenic Surgery, Shengjing Hospital, China Medical University, No. 36 \\ Sanhao Street, Heping District, Shenyang 110004, China. Email: daicl_sj@163.com.
}

Submitted Oct 19, 2018. Accepted for publication Nov 05, 2018.

doi: 10.21037/hbsn.2018.11.01

View this article at: http://dx.doi.org/10.21037/hbsn.2018.11.01

A 14-year-old girl presented with right upper abdominal swelling for two months. Physical examination revealed palpable hepatomegaly. Her laboratory analysis revealed a mild anemia (HGB: $108 \mathrm{~g} / \mathrm{L}$ ). The tests for liver function were normal. The Child-Pugh score was A grade. The tests for hepatitis $\mathrm{B}$ and $\mathrm{C}$ viruses were negative. The tests for tumor markers such as carcinoembryonic antigen (CEA), $\alpha$-fetoprotein (AFP), and cancer antigen 19-9 (CA19-9) were normal. All other laboratory results were within the normal range. Abdominal ultrasound revealed an oval isoechoic mass in the liver measuring $26.4 \mathrm{~cm} \times 25.6 \mathrm{~cm}$ $\times 12.6 \mathrm{~cm}$, which was solid, ill-defined and had rich blood flow signals. Contrast-enhanced CT and MRI of the liver demonstrated a huge hepatic mass of $14.4 \mathrm{~cm} \times 21.5 \mathrm{~cm}$ $\times 22.2 \mathrm{~cm}$, and heterogenous enhancement with central necrosis, which involved all three hepatic veins, the left portal vein and had loss of fat planes with the inferior vena cava (Figure 1A,B). Three-dimensional reconstruction technology revealed that the total hepatic volume was $6,158.3 \mathrm{~mL}$, and the tumor volume was $4,258.68 \mathrm{~mL}$. Furthermore, the standard hepatic volume was $1,105 \mathrm{~mL}$, the remnant liver volume was $1,373 \mathrm{~mL}(72 \%)$, and the indocyanine green clearance test at 15 minutes was $1.2 \%$.

The mass was a well-defined encapsulated tumor, measuring $27 \mathrm{~cm} \times 21 \mathrm{~cm} \times 11 \mathrm{~cm}$ (Figure 1C). The gross specimen consisted of an ovoid tumor, which was firm in consistency and had a pink cut surface (Figure 1D). Histologically, there was an ill-defined proliferation that mostly consisted of spindle-shaped mesenchymal cells arranged in a star-like or flaky pattern. The structure of the hepatic lobule surrounding the tumor mass was destroyed with areas of the portal fibrosis, the small bile duct hyperplasia, and the thickening of blood vessels mixed with diffusely scattered inflammatory cells. Portal venous congestion and central necrosis of the hepatic lobule was present.

On the immunohistochemistry, tumor cells revealed positive immunoreactivity for vimentin, smooth muscle actin (SMA) and Ki-67 (2\%), and negative immunoreactivity for CD34, S-100, CD99, Bcl-2, CD35, CD23 and anaplastic lymphoma kinase (ALK).

During the follow up period, no adjuvant therapy was offered. The patient had no symptoms during the 19-month follow-up and had good quality of life. During this period, ultrasound and CT revealed no signs of tumor recurrence or metastatic disease.

\section{Discussion}

Mesenchymal liver tumors are uncommonly encountered in clinical practice. These usually present with constitutional symptoms, such as abdominal pain, weight loss, weakness, loss of appetite and vomiting. Most of these tumors are suspected on radiological imaging. However, it is difficult to make an accurate diagnosis since the clinical presentation and images are non-specific. Hence, the definitive diagnosis of these tumors in most of cases is made through histopathological examination (1). In the present case, the preoperative diagnosis was primary hepatic malignant tumor.

Vimentin has been identified as a marker of epithelial mesenchymal transition (EMT), which is present in mesenchymal cells, and involved in cancer progression $(2,3)$. In the present case, the tissue stained positively for vimentin, 

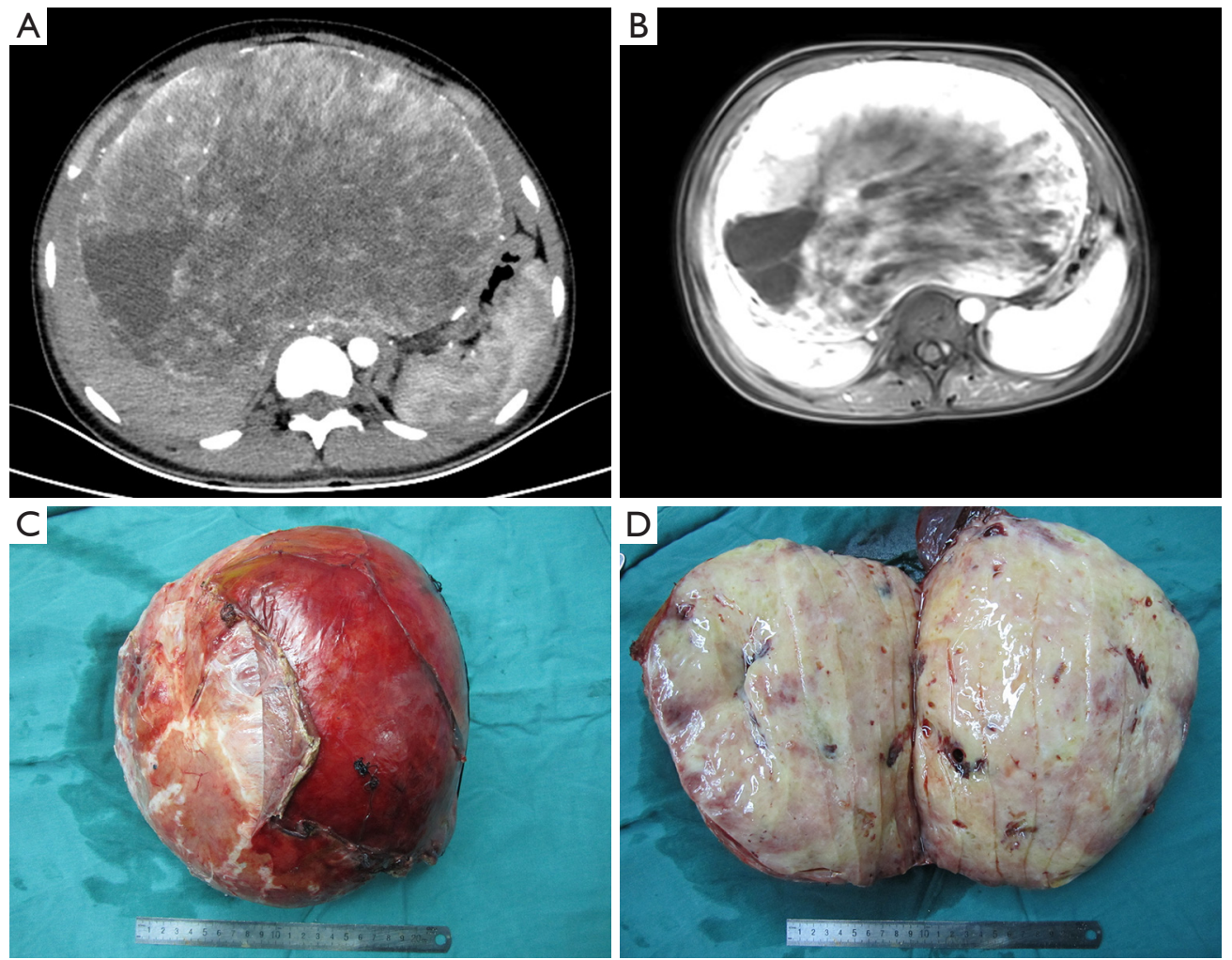

Figure 1 Imaging and gross specimen pictures. (A,B) CT and MRI demonstrates heterogenous enhancement with central necrosis; (C) the mass was a well-defined encapsulated tumor; (D) the cut surface is pink.

confirming it to be a mesenchymal tumor. Ki-67 is one of the proliferating cell nuclear antigens (4). Furthermore, it is a reliable index for evaluating cell proliferation and the malignant potential of tumors and predicting cancer recurrence $(4,5)$. The malignant potential of the index case was considered to be as low as the Ki-67 index, which was $2 \%$. In the index case, the final pathological diagnosis was established as a low-grade malignant liver mesenchymal tumor. However, hematoxylin and eosin $(\mathrm{H} \& \mathrm{E})$ staining and immunohistochemistry staining could not determine the source of the mesenchymal cells. Among various mesenchymal tumors, based on the morphological pattern on histology, the tumor in the present case appears to be most likely an inflammatory myofibroblastic tumor (IMT) or mesenchymal hamartoma (MH).

IMT and MH are rare mesenchymal tumors of the liver. Both tumors comprise of spindle-shaped mesenchymal cells, which is similar to that observed in this teenager. Furthermore, IMT, which is comprises of spindle cells, myofibroblasts, plasma cells, lymphocytes and histiocytes (2). In addition, IMT, also referred to as inflammatory myofibrohistiocytic proliferation, is a rare type of low-grade malignant mesenchymal tumor that rarely metastasizes (3). Patients usually present with a mass lesion without any specific symptoms (6). One of the characteristic features of IMT is the overexpression of ALK. Although it may not be present in all cases, ALK or ALK-1 helps in differentiating IMT from other spindle cell neoplasms (7). ALK was negative in the index case. In addition, IMTs have much more inflammatory cell infiltration, than that observed in the present case.

$\mathrm{MH}$ is the second-most common hepatic benign tumor in children. Although there are reports of its spontaneous regression, it can potentially progress to an aggressive malignant undifferentiated embryonal sarcoma (UES) (8). The most common presentation is painless abdominal distension. Histologically, MH consists of mesenchymal stellate cell proliferation in a loose mucopolysacchariderich matrix, and hepatocyte cords that surround the vessels and bile ducts. Mesenchymal collagen fibrils separate to form pseudocysts (9). In addition, as the name implies, it also contains other components, such as smooth muscles, 
adipose cells and calcification, which were less frequent in the present patient.

In conclusion, primary malignant mesenchymal tumor of the liver should be included in the differential diagnosis, when treating teenagers with an isolated liver mass. Furthermore, its long-term prognosis is unknown, and regular follow-up is essential.

\section{Acknowledgements}

None.

\section{Footnote}

Conflicts of Interest: The authors have no conflicts of interest to declare.

Informed Consent: Written informed consent was obtained from the patient for publication of this manuscript and any accompanying images.

\section{References}

1. Bhargava P, Iyer RS, Moshiri M, et al. Radiologicpathologic correlation of uncommon mesenchymal liver tumors. Curr Probl Diagn Radiol 2013;42:183-90.

Cite this article as: Zhang $\mathrm{X}$, Jia C, Zhao Y, Dai C, Xu C. A giant primary hepatic low-grade malignant mesenchymal tumor of the liver in a teenager. HepatoBiliary Surg Nutr 2018;7(6):505-507. doi: 10.21037/hbsn.2018.11.01
2. Bjelovic M, Micev M, Spica B, et al. Primary inflammatory myofibroblastic tumor of the stomach in an adult woman: a case report and review of the literature. World J Surg Oncol 2013;11:35.

3. Boudhas A, Allaoui M, El Asri F, et al. Inflammatory myofibroblastic tumor of the lacrimal gland: case report of an exceptional location. BMC Clin Pathol 2017;17:12.

4. Sun J, Li DM, Huang J, et al. The correlation between the expression of ADAM17, EGFR and Ki-67 in malignant gliomas. Eur Rev Med Pharmacol Sci 2017;21:4595-9.

5. Richardsen E, Andersen S, Al-Saad S, et al. Evaluation of the proliferation marker $\mathrm{Ki}-67$ in a large prostatectomy cohort. PLoS One 2017;12:e0186852.

6. Savvidou OD, Sakellariou VI, Papakonstantinou O, et al. Inflammatory myofibroblastic tumor of the thigh: presentation of a rare case and review of the literature. Case Rep Orthop 2015;2015:814241.

7. Hansen CC, Eisenbach C, Torres C, et al. Maxillary sinus inflammatory myofibroblastic tumors: a review and case report. Case Rep Oncol Med 2015;2015:953857.

8. Thampy R, Elsayes KM, Menias CO, et al. Imaging features of rare mesenychmal liver tumours: beyond haemangiomas. Br J Radiol 2017;90:20170373.

9. Sen D, Gulati YS, Majumder A, et al. Hepatic cystic mesenchymal hamartoma. Med J Armed Forces India 2015;71:S574-7. 